tangential electric force will be proportional to $r$; both these results are in accordance with the solution.

On this view the formation of the light quantum and its guidance is due to the magnetic force inside the atom. After it has been started on the way it should go, it will continue on that way without further guidance. A bullet has to be guided by the barrel of the rifle, but it is not necessary for the barrel to extend to the target.

Trinity Lodge,

J. J. Thomson.

Cambridge.

April 21.

${ }^{1}$ NATURE, 137, 232, Feb. 8, 1936.

NATURE, 137, 582, April 4, 1936

3 NATURE, 137, 663, April 18, 1936 .

\section{Phenomenological Theory of Supra-conductivity}

THE latest version ${ }^{1}$ of $F$. and $H$. London's phenomenological theory of supra-conductivity (which on its first appearance ${ }^{2}$ was obscured by an erroneous assumption as regards the boundary conditions ${ }^{3}$ ) can be put into a very simple form. We assume Maxwell's equations for a medium with dielectric constant $\varepsilon$ and permeability 1 (choosing the units so as to make $c=1$ and abolish odious $4 \pi$ 's) :

$$
\begin{array}{ll}
\operatorname{curl} E=-\dot{H} & (A) \quad \operatorname{curl} H=\varepsilon \dot{E}+I \\
\operatorname{div} H=0 &
\end{array}
$$

In empty space, $I=0$. In a normally conducting metal there is a current of conduction $I_{c}$ in addition to the displacement current $\varepsilon \dot{E}$ :

$$
I=I_{c}=\sigma E .
$$

The assumption for the supra-conductor is, that

$$
I=I_{c}+I_{8}=\sigma E+I_{8} .
$$

That is to say, there is a third sort of current $I_{s}$, call it the supra-current, which either is added to, or (if $\sigma=0$ ) replaces the ordinary conduction current $I_{c}$.

$I_{8}$ cannot be given in quite so simple a way as $I_{c}$, yet there is a certain analogy with the first two types of current. Let us introduce, just for the moment, the sign $I_{d}=\varepsilon \dot{E}$ for the displacement current. Then from $(A)$

$$
\operatorname{curl} \frac{I_{d}}{\varepsilon}=-\ddot{H}, \frac{\dot{I}_{d}}{\varepsilon}=\ddot{E} ;
$$

and from $(A)$ and $(C)$

$$
\operatorname{curl} \frac{I_{c}}{\sigma}=-\dot{H}, \frac{\dot{I_{c}}}{\sigma}=\dot{E}
$$

The new assumption with respect to $I_{8}$ is,

$$
\text { curl } \Lambda I_{8}=-H, \Lambda \dot{I}_{8}=E,
$$

$\Lambda$ being a constant of the material, like $\varepsilon^{-1}$ and $\sigma^{-1}$. The analogy is conspicuous. $\Lambda$ might be called the constant of supra-conductivity.

The equations $(D)$ would seem rather abundant for the only purpose of introducing the third type of current. But they contain $(A)$, which therefore can be dropped. So the full system of equations for the supra-conductor read

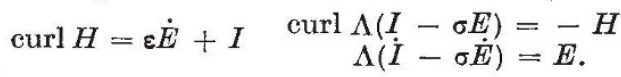

Thus there are nine equations for the nine vector components of $E, H, I$. As to the surfaces of dis- continuity, the well-known limiting conditions of Maxwell's theory $\left(E_{1}, H_{11},(\varepsilon \dot{E}+I)_{1}, H_{\perp}\right.$ continuous) have, of course, to be retained. In addition, the second curl-equation of (1) requires the continuity of $\Lambda(I-\sigma E)_{i]}$ at the surface between two different supra-conductors. (The parallel component of supracurrent will therefore have a discontinuity.)

The natural problem of initial values for the supra-conductor would be to give oneself $E$ and $I$. Equations (1) then determine the future development uniquely. If, alternatively, $E, H$ are to be given, one has to take care to choose $H$ solenoidal (div $H=0$ ), but in addition a curl-free part of $\Lambda I$ remains arbitrary.

The equations containing $\Lambda$ may be taken to state that the negative of the product $\Lambda$ by supra-current. is a suitable vector potential to represent the $E, H$. field within every coherent supra-conducting region, the scalar potential being zero. From here the theory of integration is easily developed. We shall only observe, that for the density $\rho=\varepsilon \operatorname{div} E$ we get the equation

$$
\ddot{p}+\sigma \dot{p}+\Lambda^{-1} \rho=0,
$$

which amounts virtually to $p=0$ always and every. where in the homogeneous supra-conductor. In the stationary case $(\delta / \delta t=0)$, from the last equation of (1), $E$ is zero in the supra-conductor. The $E$-lines therefore issue orthogonally from its surface, which, for the outside, acts as a surface of constant potential like with an ordinary conductor of vanishing resistance.

I consider this form of London's theory a rational heuristic starting point. So far as I can see, it is both self-consistent and without contradiction of other principles. The actual state of affairs is, of course, more complicated. It is well known that it presents phenomena of hysteresis, which cannot be embodied in a simple field theory.

24 Northmoor Road,

E. SCHRÖDINGER. Oxford.

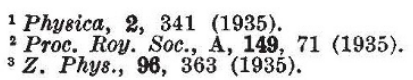

\section{Scattering of Neutrons by Protons}

IT is known that hydrogen shows a large scattering cross-section for slow neutrons. On the usual assump. tion that the forces between proton and neutron are of short range, this can only be explained if one also assumes ${ }^{1}$ that the deuteron has an excited state, real or virtual, the energy of which $\left|W_{1}\right|$ is about 50,000 e.v. This leads to the following formula for the scattering cross-section $\sigma$ of hydrogen for neutrons of energy $E$ :

$$
\sigma=\frac{h^{2}}{\pi M}\left\{\frac{3}{4} \frac{1}{W_{0}+\frac{1}{2} E}+\frac{1}{\left|W_{1}\right|+\frac{1}{2} E}\right\} .
$$

Here $h$ is Planck's constant, $\frac{1}{2} M$ is the reduced mass of the proton and neutron, and $W_{0}$ is the binding energy of the deuteron ${ }^{2,3}$ (cf. also ref. 4) $\left(W_{0}=\right.$ $2.2 \times 10^{6}$ e.v.). $\left|W_{1}\right|$ is defined by the value of $\sigma$ for $E \rightarrow 0$ (slow neutrons).

To check this formula, I have measured the mean free path in paraffin of the photo-neutrons from radiothorium and deuterium ${ }^{2}$. These neutrons are fairly homogeneous in velocity and their average energy is probably ${ }^{2,3}$ (cf. also ref. 4) about 200,000 e.v. The photo-neutron source consisted of 5 millicuries 